\title{
Trigger Factor Flexibility
}

A nuclear magnetic resonance study shows how the bacterial chaperone trigger factor can dynamically bind and release many different substrates.

\author{
Martin Gamerdinger and Elke Deuerling
}

$\mathrm{M}$ olecular chaper ones are found in all cells and are essential for maintaining a functional proteome. The main function of chaperones is to promote correct protein folding by protecting non native proteins from folding along pathways that lead to protein misfolding and aggre gation. To fulfill this task, chaperones must recognize a non native protein, transiently bind to it, and then release it at precisely the right time to allow the substrate to pro ceed with its folding course. Many but not all chaperones use adenosine $5^{\prime}$ triphosphate (ATP) to control the dynamic substrate binding and release cycle (1). On page 597 of this issue, Saio et al. (2) unravel the structural basis and underlying mechanism of action of the ATP inde pendent chaperone trigger factor (TF).

$\mathrm{TF}$ is an abundant bacterial chaperone that associates transiently with ribosomes and binds to nascent polypeptide chains. It has an extended, dragon like structure with a central body, two protruding arms, a head, and a tail region. Bound to the ribosome as a monomer by its amino terminal tail, TF leans over the ribosomal exit tunnel, thereby exposing its large interior surface speckled with multiple hydrophobic patches to the exiting nascent peptide chain (3) (see the figure). Eukaryotes use structurally different ribosome associated chaperones, but bacte rial TF is by far the best studied one and is regarded as a paradigm for ribosome bound chaperones that support the folding of newly synthesized proteins (4).

The first evidence that TF acts as a chap erone came from experiments showing that combined loss of TF and another chaperone led to synergistic defects in protein folding, resulting in global protein aggregation and decreased viability $(5,6)$. Ribosome bound

Department of Biology, University of Konstanz, 78457 Kon stanz, Germany. E mail: elke.deuerling@uni konstanz.de
TF interacts with most newly synthesized polypeptides (7) and prevents their premature folding, thereby laying the groundwork for subsequent productive folding that involves additional chaperones (8). Recent data sug gest that TF is much more versatile than ini tially thought. It can reshape and improve the folding pathway of a protein by protecting partially folded intermediates (9). Even more intriguing, TF can reverse premature fold ing by facilitating unfolding of preformed structures in nascent polypeptides, allowing the nascent peptide to reenter the productive folding path (10).

Despite comprehensive analyses of TF over many years, the molecular details of its versatile chaperone activity were not under stood. Using sophisticated nuclear mag netic resonance techniques, Saio et al. have now determined the structure and dynamics of purified TF as it interacts with unfolded model substrates in solution, thereby disclos ing its mechanism of action in great detail.

The authors show that TF forms a binding scaffold with four distinct substrate binding sites that are distributed along its inner surface (see the figure). The four bind ing sites can be used in a vari able order, and not all sites are always occupied by sub strates. The binding sites con tain nonpolar residues form ing hydrophobic pockets that bind to hydrophobic peptide stretches of 6 to 10 residues in substrate proteins. The binding sites have a flexible local architecture that allows interaction with a large and diverse population of pep tide stretches with unrelated primary sequences. Further more, polar residues next to the hydrophobic binding sites can form hydrogen bonds with the substrate, probably to enhance affinity and navigate binding. This high degree of plasticity of its binding sur faces explains how TF can serve a large pool of nascent substrates.

How does TF affect fold ing of substrates using this highly flexible binding scaffold? Using all of its four binding sites, TF can directly bind up to 50 substrate residues. The hydropho bic peptide stretches bound by TF are sepa rated by linker regions that remain unbound and may even loop outward (see the figure). Moreover, supporting earlier findings (11), Saio et al. show that multiple TF molecules can bind simultaneously to a substrate. This multistage chaperone mechanism enables TF to retain large polypeptides in an unfolded state and protect them from aggregation by shielding their exposed hydrophobic regions. The multivalent binding of TF to its substrate may even provide enough binding energy to unfold misfolded peptide structures of low thermodynamic stability.

TF binds with low affinity to individ ual substrate binding sites, but the affin ity gradually increases with the number of bound segments. Substrate binding to the binding sites is highly dynamic and the res idence time is short $\left(1 \mathrm{~ms}\right.$ at $\left.25^{\circ} \mathrm{C}\right)$. Even when TF fully engages its unfolded protein substrate by using all of its available bind ing sites, the complex remains dynamic, with fast association and dissociation rates. This 
highly dynamic substrate engagement is very important during protein synthesis, allow ing TF to accommodate the permanently changing composition of binding sites in the nascent polypeptide that grows by up to 20 amino acid residues per second.

Saio et al.'s study provides a milestone in understanding how TF performs its dynamic and multifaceted functions in the cell. The work will pave the way for further exciting advances in understanding the plasticity of this and other ATP independent chaperones. However, several important questions remain to be answered about the activity of the TF chaperone. For example, Saio et al. investi gated purified TF, which was not in complex with the ribosome, and it thus remains to be shown whether the binding sites of ribosome tethered TF are similar. Three binding sites are located in the central body and one in the head domain, but no binding site is found in the amino terminal tail that tethers TF to the ribosome (see the figure). TF undergoes some structural changes upon binding to ribosomes (11) and may thus expose other or additional binding sites.

Another open question is how the dynamic association of one or perhaps more TF mol ecules with nascent polypeptides is coordi nated with the activity of other chaperones that bind to nascent proteins in later stages of protein synthesis (3). And finally, TF that is not bound to ribosomes can form dimers that can encapsulate small native like proteins using primarily polar contacts. This TF activ ity may promote the assembly of substrates into large complexes (12). Further analyses are required to understand how TF can switch between hydrophobic and hydrophilic bind ing modes for substrates.

\section{References}

1. Y. E. Kim et al. Annu. Rev. Biochem. 82, 323 (2013).

2. T. Saio et al., Science 344, 1250494 (2014); 10.1126/ science.1250494.

3. L. Ferbitz et al., Nature 431, 590 (2004).

4. A. Hoffmann, B. Bukau, G. Kramer, Biochim. Biophys. Acta 1803, 650 (2010).

5. E. Deuerling et al., Nature 400, 693 (1999).

6. S. A. Teter et al., Cell 97, 755 (1999).

7. E. Oh et al., Cell 147, 1295 (2011).

8. V. R. Agashe et al., Cell 117, 199 (2004).

9. A. Mashaghi et al., Nature 500, 98 (2013).

10. A. Hoffmann et al., Mol. Cell 48, 63 (2012).

11. C. M. Kaiser et al., Nature 444, 455 (2006).

12. E. Martinez Hackert, W. A. Hendrickson, Cell 138, 923 (2009) 